\title{
THE BASICS OF DYNAMICALLY CORRECTING THE SENSITIVITY COEFFICIENTS OF A GROUP OF SINGLE-TYPE SENSORS FOR UNIFORM AND TRIANGULAR DISTRIBUTION LAWS
}

Предполагается, что на этапе эксплуатации существует возможность производить измерение изотропного фона. Выполняя сортировку измеренных значений в группе однотипных датчиков, в случае одинаковости их чувствительности получаем для каждого датчика равномерную картину распределения по позициям сортированного списка. В случае дрейфа чувствительности одного датчика возникает дисбаланс: измеренные значения от этого датчика оказываются в одной половине чаще, чем в другой.

Представлены формулы и результаты вычислений, устанавливающие зависимость дисбаланса от величины дрейфа одного датчика в качестве основы для динамической корректировки коэффициентов чувствительности однотипных датчиков. Использованы равномерный и треугольный законы распределения, а также предложена возможность применения приближенных зависимостей для других типов распределения.

ДАТЧИКИ; ЧУВСТВИТЕЛЬНОСТЬ; ДРЕЙФ; КОРРЕКТИРОВКА; ТОЧНОСТЬ; ЖИВУЧЕСТЬ; САМОВОССТАНОВЛЕНИЕ.

It is assumed that there is an opportunity of measuring the isotropic background at an operational stage. Sorting the measured values in a group of sensors of the same type, in case of similarity of their sensitivity we obtain for each sensor a uniform picture of distribution over the positions of the sorted list. In case of a sensitivity drift of one sensor there is an imbalance: the measured values from this sensor appear in one half more often than in another.

The paper presents formulas and results of the calculations establishing the dependence of the imbalance on the size of the drift of one sensor as a basis for dynamically correcting the sensitivity coefficients of the same sensors. Uniform and triangular distribution laws are used, and also the possibility of using approximate dependences for other types of distribution is offered.

SENSORS; SENSITIVITY; DRIFT; ADJUSTMENT; ACCURACY; SURVIVABILITY; SELFRESTORATION.

Важнейшей частью современных робототехнических и человеко-машинных систем являются датчики (сенсоры) различных типов. При этом высокие требования к живучести и точности измерений в сочетании с невозможностью или затруднительностью 
участия человека в выполнении ремонтновосстановительных работ в экстремальных условиях эксплуатации приводят к необходимости интеллектуализации датчиков. В [1] указано, что, в отличие от адаптивного датчика, обладающего свойствами автоматической настройки своих параметров в зависимости от текущих условий, интеллектуальный датчик дополнительно должен осуществлять: автоматическую коррекцию погрешности, появившейся в результате воздействия влияющих величин и/или старения компонентов; самовосстановление при возникновении единичного дефекта в датчике; самообучение.

Как известно, живая природа предоставляет нам примеры множественности рецепторов в составе глаза и уха. Сенсоры технических систем во многом похожи на органы зрения и слуха. Это сходство может проявляться по-разному, но в рамках темы данной статьи уместно обратить внимание на следующие примеры применения набора однотипных датчиков:

- акустическая система, содержащая ряд микрофонов вокруг башни в составе боевого робота «Скорпион» [2], обеспечивает возможность моментально и весьма точно определять направление на источник звука (выстрела), чтобы соответственно направлять ствол пушки;

- измеритель мощности дозы (ИМД) [3-5], содержащий ряд сенсоров (счетчиков Гейгера) вокруг цилиндра из вольфрамового сплава, также предоставляет пользователю угол направления на источник радиационного излучения.

Характерной особенностью систем такого вида является необходимость одинаковой чувствительности датчиков. Между тем параметры различных производимых счетчиков регистрации излучения представлены в [6]. При этом в графе «Чувствительность» указано, например: 60-75 имп/мкР для счетчика «Гамма-7»; 285-385 имп/мкР для счетчика «Гамма-8»; 31-39 имп/мкР для счетчика «Гамма-10». Как видно, каждая модель допускает изначальный разброс около $25 \%$. В связи с этим выполняется настройка параметров программного обеспечения (коэффициентов чувствительно- сти) в устройствах радиационного контроля прежде всего на этапе изготовления, а также периодически и на этапе эксплуатации при регламентных работах, с использованием эталонного источника.

Но если мы говорим об актуальности интеллектуализации сенсорных систем, то поставим задачу динамической корректировки коэффициентов чувствительности, т. е. непосредственно в ходе рабочего функционирования на этапе эксплуатации, без эталонного источника.

Такая возможность существует и является благоприятной в той мере, в какой допускается считать, что фон, накопленный за длительное время в промежутках между ситуациями наличия источников, обладает свойством изотропности.

Актуальность совершенствования средств радиационного контроля указана, например, в [7]. При этом предполагается использование мобильных наземных, воздушных и подводных робототехнических средств. Конечно, они могут быть и стационарными, установленными в удаленных, опасных и труднодоступных местах. Во всех вариантах более предпочтительными являются интеллектуальные измерительные средства, обладающие свойством самовосстановления.

\section{Постановка задачи и базовые формулы}

Считаем, что нам задана зависимость математического ожидания измеренного значения от параметров настройки датчика. Соответственно имеем возможность манипулировать смещениями. В частности, в составе программного обеспечения ИМД для каждого датчика предусмотрен коэффициент чувствительности, номинальное значение которого равно единице.

Дрейфу подвержены все датчики, но в интервале между сеансами пересчета коэффициентов чувствительности чрезмерно увеличившийся дрейф наиболее вероятен только у одного датчика. Поэтому в рамках данной статьи в используемой математической модели предполагается, что дрейфу подвержен только один датчик.

Цель статьи - получение формул, на основе которых будет обеспечена возмож- 
ность вычислить величину дрейфа датчика, чтобы затем произвести пересчет его параметров настройки (коэффициента чувствительности).

Для этого в программном обеспечении изделия должно быть предусмотрено формирование исходных данных следующим образом.

Проверяя отсутствие источника излучения (отсутствие завышенной неравномерности результатов измерений, поступающих от датчиков), выполняем накопление фона в течение очередного интервала времени (такта). На каждом такте выполняем сортировку значений, полученных от всех датчиков. Формируем массив счетчиков $B_{i, j}-$ количество случаев попадания $i$-го датчика в $j$-ю позицию сортированного списка значений. Отсчет индексов производим от нуля.

Пусть $M-$ количество тактов; $N-$ количество счетчиков. Очевидно, что для каждого значения $i$ соблюдается равенство

$$
M=\sum_{j=0}^{N-1} B_{i, j}
$$

Для каждого датчика вычисляем нормированные суммы на половине счетчиков:

$Q_{i}=\frac{1}{M} \sum_{j=0}^{\frac{N}{2}-1} B_{i, j}$ при четном количестве $N$;

$Q_{i}=\frac{1}{M}\left(\frac{1}{2} B_{i, \frac{N-1}{2}}+\sum_{j=0}^{\frac{N-1}{2}-1} B_{i, j}\right) \quad$ при нечетном количестве $N$.

Каждую такую сумму $Q_{i}$ будем называть дисбалансом. При отсутствии дрейфа (в исходном состоянии) она равна $1 / 2$, а при увеличении дрейфа до полной ширины статистического разброса дисбаланс становится равным единице.

Понятно, что дрейфующий датчик приобретает наибольший дисбаланс, а остальные датчики приобретают меньший (равномерно распределенный между ними) дисбаланс противоположной направленности.

Пусть задана функция $p(a, x)$ - плотность вероятности распределения измеряемой величины $x$ в области значений, ширина которой характеризуется параметром $a$.
При этом без ограничения общности для удобства вычислений можно считать, что среднее значение равно нулю.

Обозначим $L$ величину дрейфа в сторону занижения. Это значит, что плотность вероятности приобретает вид $p(a, x+L)$.

Поставленная цель статьи формализуется как выявление зависимости $Q(L)$, соответствующей одному дрейфующему датчикy.

Обозначим и интегральную функцию вероятности распределения:

$$
P(a, X)=\int_{-\infty}^{X} p(a, x) d x .
$$

Запишем вероятность попадания дрейфующего датчика в $j$-ю позицию:

$$
\begin{aligned}
& q(a, L, N, j)=\frac{(N-1) !}{j !(N-1-j) !} \int_{-\infty}^{\infty} P(a, X)^{j} \times \\
& \quad \times(1-P(a, X))^{N-1-j} p(a, X+L) d X .
\end{aligned}
$$

Здесь в подынтегральном выражении первый множитель соответствует $j$ датчикам, у которых измеренное значение оказалось меньше, чем $X$; второй множитель соответствует $N-1-j$ датчикам, у которых измеренное значение оказалось больше, чем $X$; а третий множитель определяет вероятность того, что дрейфующий датчик дает значение в интервале от $X$ до $X+d X$. Комбинаторный коэффициент учитывает варианты перестановок всех датчиков, кроме дрейфующего.

Кстати, уместно заметить, что формула (2) определяет вероятность того, что значение от дрейфующего датчика оказалось $j$-й порядковой статистикой. В отличие от формул, имеющихся, например, в [8] или [9], записанных для одинаковых датчиков, для варианта с дрейфующим датчиком каждый момент $j$-й порядковой статистики может быть записан в виде суммы трех величин. При этом (2) является одной из этих трех величин для момента порядка нуля.

Но в данной статье задача ставится иначе: вычисление интеграла (2) и суммирование по половине позиций.

При четном $N$ :

$$
Q(a, L, N)=\sum_{j=0}^{N / 2-1} q(a, L, N, j) .
$$


При нечетном $N$ имеется серединное место, которое надо делить пополам между двумя половинами набора счетчиков:

$$
\begin{gathered}
Q(a, L, N)=\frac{1}{2} q\left(a, L, N, \frac{N-1}{2}\right)+ \\
+\sum_{j=0}^{(N-1) / 2-1} q(a, L, N, j) .
\end{gathered}
$$

Обозначим серединный индекс: при четном $N$

$$
n=\frac{N}{2}-1
$$

при нечетном $N$

$$
n=\frac{N-1}{2} .
$$

Обозначим серединный множитель: при четном $N$

$$
\delta(j)=1 ;
$$

при нечетном $N$

$$
\begin{gathered}
\delta(j)=1, \text { при } j \neq n ; \\
\delta(n)=1 / 2 .
\end{gathered}
$$

В этих обозначениях формулы (3) и (4) выглядят как единая формула:

$$
Q(a, L, N)=\sum_{j=0}^{n} \delta(j) q(a, L, N, j) .
$$

\section{Вариант равномерного распределения}

Обозначим $(-a, a)-$ интервал случайного разброса значений, получаемых от каждого датчика.

Плотность вероятности распределения имеет вид:

$$
\begin{aligned}
& p(a, x)=1 /(2 a) \text { при }-a \leq x \leq a ; \\
& p(a, x)=0 \text { при } x<-a \text { и при } x>a .
\end{aligned}
$$

Дисперсия:

$$
2 \frac{1}{2 a} \int_{0}^{a} x^{2} d x=\left[\frac{x^{3}}{3 a}\right]_{0}^{a}=\frac{a^{2}}{3} .
$$

Интегралы пропорциональны интервалам интегрирования:

$$
\begin{gathered}
P(a, X)=\int_{-a}^{X} d x /(2 a)=(a+X) /(2 a) \\
1-P(a, X)=\int_{X}^{a} d x /(2 a)=(a-X) /(2 a)
\end{gathered}
$$

Если дрейфующий датчик дает значение меньше, чем $-a$, то оно заведомо попадает в нулевую позицию. Вероятность такого события $L /(2 a)$.

Оставшаяся часть интервала возможных значений дрейфующего датчика от $-a$ до $a-L$ подлежит быть интервалом интегрирования для формулы (2), которая после подстановки (11) и (12) приобретает следующий вид:

$$
\begin{gathered}
q(a, L, N, j)=\frac{(N-1) !}{j !(N-1-j) !(2 a)^{N}} \times \\
\times \int_{-a}^{a-L}(a+X)^{j}(a-X)^{N-1-j} d X ;
\end{gathered}
$$

при $j=0$ имеем:

$$
q(a, L, N, 0)=\frac{1}{N}\left(1-\left(\frac{L}{2 a}\right)^{N}\right) ;
$$

при $j>0$, интегрируя (13) «по частям», получаем:

$$
\begin{gathered}
q(a, L, N, j)=\frac{(N-1) !}{(2 a)^{N}} \times \\
\times\left(\int_{-a}^{a-L} \frac{(a+X)^{j-1}(a-X)^{N-j}}{(j-1) !(N-j) !} d X-\right. \\
\left.-\frac{(2 a-L)^{j} L^{N-j}}{j !(N-j) !}\right)
\end{gathered}
$$

В полученном выражении (15) просматривается выражение (13) с предшествующим значением индекса $j$, поэтому оно приобретает рекурсивный вид:

$$
\begin{gathered}
q(a, L, N, j)=q(a, L, N, j-1)- \\
-\frac{(N-1) !}{(2 a)^{N}} \frac{(2 a-L)^{j} L^{N-j}}{j !(N-j) !} .
\end{gathered}
$$

Цикл вычисления суммы (3) или (4) удобно выполнять в порядке убывания индекса $j$. На каждом шаге получаем возможность благодаря формуле (16) избавиться от одного знака интеграла и в качестве следствия приведения подобных членов добавить множитель $(N / 2-j)$ к очередному слагаемому $q(a, L, N, j)$. На завершающем шаге работает формула (14), свободная от знака интеграла и от рекурсивной связи.

В итоге получаем следующую формулу: 


$$
\begin{gathered}
Q(a, L, N)=\frac{L}{2 a}-\frac{(N-1) !}{(2 a)^{N}} \times \\
\times \sum_{j=1}^{n} \frac{(N / 2-j)(2 a-L)^{j} L^{N-j}}{j !(N-j) !}+ \\
+\frac{1}{2}\left(1-\left(\frac{L}{2 a}\right)^{N}\right) .
\end{gathered}
$$

При нечетном $N$ дробь $N / 2$ в формуле (17) так и должна быть числом с половинкой, которая происходит от коэффициента $1 / 2$ в формуле (4).

Обозначив $s=L /(2 a)$, перепишем формулу (17) в более удобном виде:

$$
\begin{gathered}
Q(s, N)=s+\frac{1}{2}\left(1-s^{N}\right)- \\
-\sum_{j=1}^{n} \frac{(N / 2-j)(N-1) !(1-s)^{j} s^{N-j}}{j !(N-j) !} .
\end{gathered}
$$

Как и следовало ожидать, при любом $N$ соблюдаются равенства:

$$
Q(0, N)=\frac{1}{2} ; \quad Q(1, N)=1 .
$$

Существует возможность дальнейшего преобразования формулы (18) следующим образом. Первый шаг начинается с преобразования множителя $N / 2-j$ :

$$
\begin{gathered}
Q(s, N)=\frac{1}{2}+s-\frac{1}{2} s^{N}- \\
-\frac{1}{2} \sum_{j=1}^{n} \frac{(N-j-j)(N-1) !(1-s)^{j} s^{N-j}}{j !(N-j) !} .
\end{gathered}
$$

Это позволяет нам разбить сумму на две суммы:

$$
\begin{gathered}
Q(s, N)=\frac{1}{2}+s-\frac{1}{2} s^{N}- \\
-\frac{1}{2} \sum_{j=1}^{n} \frac{(N-1) !(1-s)^{j} s^{N-j}}{j !(N-j-1) !}+ \\
+\frac{1}{2} \sum_{j=1}^{n} \frac{(N-1) !(1-s)^{j} s^{N-j}}{(j-1) !(N-j) !} .
\end{gathered}
$$

$\mathrm{K}$ первой сумме добавляем слагаемое, соответствующее значению индекса $j=0$, а во второй сумме выделяем множитель $1-s$ и переобозначаем индекс $j$ путем смещения на единицу:

$$
Q(s, N)=\frac{1}{2}+s-\frac{1}{2} \sum_{j=0}^{n} \frac{(N-1) !(1-s)^{j} s^{N-j}}{j !(N-j-1) !}+
$$

$$
+\frac{1-s}{2} \sum_{j=0}^{n-1} \frac{(N-1) !(1-s)^{j} S^{N-1-j}}{j !(N-1-j) !} .
$$

Первая сумма в отличие от второй имеет $n$-й элемент, который надо записать отдельно.

Вторую сумму в этом выражении можно разбить на две суммы, одна из которых совпадает с первой суммой без $n$-го элемента:

$$
\begin{gathered}
\frac{1}{2} \sum_{j=0}^{n-1} \frac{(N-1) !(1-s)^{j} S^{N-1-j}}{j !(N-1-j) !}- \\
-\frac{1}{2} \sum_{j=0}^{n-1} \frac{(N-1) !(1-s)^{j} s^{N-j}}{j !(N-1-j) !} .
\end{gathered}
$$

Поэтому в следующем шаге преобразований получаем:

$$
\begin{aligned}
Q(s, N) & =\frac{1}{2}+s-\frac{(N-1) !(1-s)^{n} s^{N-n}}{2 n !(N-n-1) !}- \\
& -\sum_{j=0}^{n-1} \frac{(N-1) !(1-s)^{j} s^{N-j}}{j !(N-j-1) !}+ \\
+ & \frac{1}{2} \sum_{j=0}^{n-1} \frac{(N-1) !(1-s)^{j} s^{N-1-j}}{j !(N-1-j) !} .
\end{aligned}
$$

В этой формуле суммы отличаются друг от друга только множителями $s$ и $1 / 2$, поэтому ее можно преобразовать к следующему окончательному виду с одной суммой:

$$
\begin{aligned}
Q(s, N)= & \frac{1}{2}+s-\frac{(N-1) !(1-s)^{n} s^{N-n}}{2 n !(N-n-1) !}+ \\
& +\left(\frac{1}{2}-s\right) W(s, N) .
\end{aligned}
$$

Здесь обозначено:

$$
W(s, N)=\sum_{j=0}^{n-1} \frac{(N-1) !(1-s)^{j} s^{N-1-j}}{j !(N-j-1) !} .
$$

Полученная формула (20) интересна тем, что при $s=1 / 2$ избавляет нас от необходимости вычислять сумму $W(s, N)$. Иначе говоря, когда дрейф датчика достигает половины ширины области случайного разброса измеряемой величины, имеем весьма лаконичную формулу дисбаланса счетчиков попаданий результатов измерений от дрейфующего датчика в позиции сортированного списка результатов измерений от всех датчиков. 
При четном $N$, учитывая (5), из (20) получаем:

$$
Q\left(\frac{1}{2}, N\right)=1-\frac{(N-1) !}{2^{N+1}\left(\frac{N}{2}-1\right) !\left(\frac{N}{2}\right) !} .
$$

При нечетном $N$, учитывая (6), из (20) получаем:

$$
Q\left(\frac{1}{2}, N\right)=1-\frac{(N-1) !}{2^{N+1}\left(\frac{N-1}{2}\right) !\left(\frac{N-1}{2}\right) !}
$$

Нетрудно убедиться, что (22) и (23) дают одинаковый результат для четного и для большего следующего нечетного $N$. Будем называть это эффектом нейтральности серединного элемента. Получается так, что в формуле (4) слагаемое с коэффициентом $1 / 2$ необходимо, но не добавляет нам информативности.

Чтобы лучше представить характер функции $Q(s, N)$, рассмотрим также ее производную:

$$
Q_{s}(s, N)=1-\frac{(N-1) !\left((N-n)(1-s)^{n} s^{N-1-n}-n(1-s)^{n-1} s^{N-n}\right)}{2 n !(N-n-1) !}+\left(\frac{1}{2}-s\right) W_{s}(s, N)-W(s, N) \text {. }
$$

Каждое слагаемое (кроме слагаемого с индексом 0) суммы $W_{s}(s, N)$ по правилу дифференцирования произведения распадается на два слагаемых, поэтому теперь имеем дело с двумя суммами:

$$
\begin{gathered}
W_{s}(s, N)=\sum_{j=0}^{n-1} \frac{(N-1) !(1-s)^{j} s^{N-2-j}}{j !(N-2-j) !}- \\
-\sum_{j=1}^{n-1} \frac{(N-1) !(1-s)^{j-1} s^{N-1-j}}{(j-1) !(N-j-1) !} .
\end{gathered}
$$

В полученной формуле (25) для второй суммы делаем замену индекса путем смещения на единицу и обнаруживаем, что она отличается от первой суммы только слагаемым с индексом $n-1$. Поэтому получаем:

$$
W_{s}(s, N)=\frac{(N-1) !(1-s)^{n-1} s^{N-1-n}}{(n-1) !(N-1-n) !} .
$$

В частности, по формуле (24) с учетом формул (21) и (26) для любого $N$ получаем:

$$
Q_{t}(0, N)=1 ; Q_{t}(1, N)=0 .
$$

Рассмотрим также случай $s=1 / 2$ :

$$
\begin{gathered}
Q_{t}\left(\frac{1}{2}, N\right)=1-\frac{(N-1) !(N-n-n)}{2^{N} n !(N-n-1) !}- \\
-\frac{1}{2^{N-1}} \sum_{j=0}^{n-1} \frac{(N-1) !}{j !(N-j-1) !}
\end{gathered}
$$

С учетом свойства симметричности биномиального ряда, получаемого разложением выражения $(1+1)^{N-1}$ [10, с. 70-72], нетрудно записать эту сумму через половину от величины $2^{N-1}$ и убедиться, что для любого $N$ соблюдается равенство:

$$
Q_{t}\left(\frac{1}{2}, N\right)=\frac{1}{2} .
$$

Таким образом, полученные формулы (общие (18), (20), (24) и частные (19), (22), (23), (27), (28)) предоставляют полную картину зависимости дисбаланса сортированных счетчиков от величины дрейфа чувствительности одного измерителя для случая равномерного распределения измеряемой случайной величины.

\section{Треугольное распределение}

Функция плотности вероятности имеет вид:

$p(a, x)=(a+x) /\left(a^{2}\right)$ при $-a \leq x \leq 0 ;$

$p(a, x)=(a-x) /\left(a^{2}\right)$ при $0 \leq x \leq a$;

$p(a, x)=0$ при $x<-a$ и при $x>a$.

Интегральная функция вероятности:

$P(a, x)=0$ при $x<-a$;

$P(a, x)=\frac{1}{2}+\frac{x}{a}+\frac{x^{2}}{2 a^{2}}=\frac{1}{2}\left(1+\frac{x}{a}\right)^{2}$

при $-a \leq x \leq 0$;

$P(a, x)=\frac{1}{2}+\frac{x}{a}-\frac{x^{2}}{2 a^{2}}=1-\frac{1}{2}\left(1-\frac{x}{a}\right)^{2}$

при $0 \leq x \leq a$;

$P(a, x)=1$ при $x>a$.

Дисперсия треугольного распределения:

$$
2 \int_{0}^{a} \frac{a-x}{a^{2}} x^{2} d x=2\left[\frac{x^{3}}{3 a}-\frac{x^{4}}{4 a^{2}}\right]_{0}^{a}=\frac{a^{2}}{6} .
$$

Угловатость функции плотности распределения порождает необходимость, применяя формулу (2), рассматривать по отдельности интервалы наложения восходящих и 
нисходящих участков функции дрейфующего датчика и функции остальных датчиков.

$$
\begin{gathered}
\text { Вариант } \mathbf{0} \leq \boldsymbol{L} \leq \boldsymbol{a} \\
+\sum_{j=0}^{n} \frac{\delta(j)(N, N)=\frac{1}{2}\left(\frac{L}{a}\right)^{2}+}{j !(N-1-j) !} \int_{-a}^{-L}\left(\frac{1}{2}\right)^{j}\left(1+\frac{x}{a}\right)^{2 j} \times \\
\times\left(1-\frac{1}{2}\left(1+\frac{x}{a}\right)^{2}\right)^{N-1-j} \frac{a+L+x}{a^{2}} d x+ \\
+\sum_{j=0}^{n} \frac{\delta(j)(N-1) !}{j !(N-1-j) !} \int_{-L}^{0}\left(\frac{1}{2}\right)^{j}\left(1+\frac{x}{a}\right)^{2 j} \times \\
\times\left(1-\frac{1}{2}\left(1+\frac{x}{a}\right)^{2}\right)^{N-1-j} \frac{a-L-x}{a^{2}} d x+ \\
+\sum_{j=0}^{n} \frac{\delta(j)(N-1) !}{j !(N-1-j) !} \int_{0}^{a}\left(\frac{1}{2}\right)^{N-1-j}\left(1-\frac{x}{a}\right)^{2(N-1-j)} \times \\
\times\left(1-\frac{1}{2}\left(1-\frac{x}{a}\right)^{2}\right)^{j} \frac{a-L-x}{a^{2}} d x .
\end{gathered}
$$

Первое слагаемое соответствует случаям, когда измерения от дрейфующего датчика оказались в интервале от $-a-L$ до $-a$, при этом они заведомо попадают в крайнюю позицию сортированного списка. Каждая из трех сумм интегралов соответствует случаям, когда измерение от дрейфующего датчика попадает в интервал, указанный в качестве границ интегрирования.

Обозначим $t=x / a ; z=L / a$. При $0 \leq z \leq 1$ перепишем полученную формулу:

$$
\begin{gathered}
Q(z, N)=\frac{1}{2} z^{2}+ \\
+\sum_{j=0}^{n} \frac{\delta(j)(N-1) !}{j !(N-1-j) !} \int_{-1}^{-z}\left(\frac{1}{2}\right)^{j}(1+t)^{2 j} \times \\
\times\left(1-\frac{1}{2}(1+t)^{2}\right)^{N-1-j}(1+z+t) d t+ \\
+\sum_{j=0}^{n} \frac{\delta(j)(N-1) !}{j !(N-1-j) !} \int_{-z}^{0}\left(\frac{1}{2}\right)^{j}(1+t)^{2 j} \times \\
\times\left(1-\frac{1}{2}(1+t)^{2}\right)^{N-1-j}(1-z-t) d t+
\end{gathered}
$$

$$
\begin{gathered}
+\sum_{j=0}^{n} \frac{\delta(j)(N-1) !}{j !(N-1-j) !} \int_{0}^{1-z}\left(\frac{1}{2}\right)^{N-1-j}(1-t)^{2(N-1-j)} \times \\
\times\left(1-\frac{1}{2}(1-t)^{2}\right)^{j}(1-z-t) d t .
\end{gathered}
$$

Сделаем замену переменной $t$ путем смещения на единицу, вынесем степень от 1/2:

$$
\begin{gathered}
Q(z, N)=\frac{1}{2} z^{2}+ \\
+\left(\frac{1}{2}\right)^{N-1} \sum_{j=0}^{n} \frac{\delta(j)(N-1) !}{j !(N-1-j) !} \times \\
\times \int_{0}^{1-z} t^{2 j}\left(2-t^{2}\right)^{N-1-j}(z+t) d t+ \\
+\left(\frac{1}{2}\right)^{N-1} \sum_{j=0}^{n} \frac{\delta(j)(N-1) !}{j !(N-1-j) !} \times \\
\times \int_{1-z}^{1} t^{2 j}\left(2-t^{2}\right)^{N-1-j}(2-z-t) d t+ \\
+\left(\frac{1}{2}\right)^{N-1} \sum_{j=0}^{n} \frac{\delta(j)(N-1) !}{j !(N-1-j) !} \times \\
\times \int_{z}^{1} t^{2(N-1-j)}\left(2-t^{2}\right)^{j}(t-z) d t .
\end{gathered}
$$

Как видно, в данном выражении присутствуют четыре типа интегралов:

$$
\begin{aligned}
U\left(t_{1}, t_{2}\right) & =\left(\frac{1}{2}\right)^{N-1} \sum_{j=0}^{n} \frac{\delta(j)(N-1) !}{j !(N-1-j) !} \times \\
& \times \int_{t_{1}}^{t_{2}} t^{2 j}\left(2-t^{2}\right)^{N-1-j} d t ; \\
V\left(t_{1}, t_{2}\right) & =\left(\frac{1}{2}\right)^{N-1} \sum_{j=0}^{n} \frac{\delta(j)(N-1) !}{j !(N-1-j) !} \times \\
& \times \int_{t_{1}}^{t_{2}} t^{2 j}\left(2-t^{2}\right)^{N-1-j} t d t ; \\
G\left(t_{1}, t_{2}\right) & =\left(\frac{1}{2}\right)^{N-1} \sum_{j=0}^{n} \frac{\delta(j)(N-1) !}{j !(N-1-j) !} \times \\
& \times \int_{t_{1}}^{t_{2}} t^{2(N-1-j)}\left(2-t^{2}\right)^{j} d t ; \\
H\left(t_{1}, t_{2}\right) & =\left(\frac{1}{2}\right)^{N-1} \sum_{j=0}^{n} \frac{\delta(j)(N-1) !}{j !(N-1-j) !} \times \\
& \times \int_{t_{1}}^{t_{2}} t^{2(N-1-j)}\left(2-t^{2}\right)^{j} t d t .
\end{aligned}
$$


Каждый из рядов (30) и (32) содержит верхнюю или нижнюю половину полного биномиального ряда $\left(t^{2}+\left(2-t^{2}\right)\right)^{N-1}$. Поэтому для любого $N$ :

$$
U\left(t_{1}, t_{2}\right)+G\left(t_{1}, t_{2}\right)=t_{2}-t_{1} .
$$

Аналогично заметим, что каждый из рядов (31) и (33) содержит верхнюю или нижнюю половину полного такого же биномиального ряда с дополнительным множителем: $t\left(t^{2}+\left(2-t^{2}\right)\right)^{N-1}$. Поэтому для любого $N$ :

$$
V\left(t_{1}, t_{2}\right)+H\left(t_{1}, t_{2}\right)=\frac{1}{2}\left(t_{2}^{2}-t_{1}^{2}\right) .
$$

Тождества (34) и (35) полезны, например, для проверки правильности программирования.

С обозначениями (30)-(33) вычисляемый дисбаланс приобретает вид:

$$
\begin{gathered}
Q(z, N)=\frac{1}{2} z^{2}+z U(0,1-z)+V(0,1-z)+ \\
+(2-z) U(1-z, 1)-V(1-z, 1)- \\
-z G(z, 1)+H(z, 1) .
\end{gathered}
$$

В частности:

$$
\begin{aligned}
& Q(0, N)=V(0,1)+H(0,1)=\frac{1}{2} \\
& Q(1, N)=\frac{1}{2}+U(0,1)-V(0,1) .
\end{aligned}
$$

Применяя известные правила вычисления производных по верхнему или нижнему пределам интегрирования, запишем также производную:

$$
\begin{gathered}
Q_{z}(z, N)=z+U(0,1-z)-U(1-z, 1)+ \\
+2(1-z) U^{\bullet}(1-z)-2 V^{\bullet}(1-z)-G(z, 1)+ \\
+z G^{\bullet}(z)-H^{\bullet}(z) .
\end{gathered}
$$

Здесь обозначены подынтегральные функции:

$$
\begin{gathered}
U^{\bullet}(t)=\left(\frac{1}{2}\right)^{N-1} \times \\
\times \sum_{j=0}^{n} \frac{\delta(j)(N-1) !}{j !(N-1-j) !} t^{2 j}\left(2-t^{2}\right)^{N-1-j} ; \\
V^{\bullet}(t)=\left(\frac{1}{2}\right)^{N-1} \times \\
\times \sum_{j=0}^{n} \frac{\delta(j)(N-1) !}{j !(N-1-j) !} t^{2 j+1}\left(2-t^{2}\right)^{N-1-j}
\end{gathered}
$$

$$
\begin{gathered}
G^{\bullet}(t)=\left(\frac{1}{2}\right)^{N-1} \times \\
\times \sum_{j=0}^{n} \frac{\delta(j)(N-1) !}{j !(N-1-j) !} t^{2(N-1-j)}\left(2-t^{2}\right)^{j} \\
H^{\bullet}(t)=\left(\frac{1}{2}\right)^{N-1} \times \\
\times \sum_{j=0}^{n} \frac{\delta(j)(N-1) !}{j !(N-1-j) !} t^{2(N-1-j)+1}\left(2-t^{2}\right)^{j} .
\end{gathered}
$$

Нетрудно убедиться, что для любого $N$ :

$$
\begin{aligned}
& U^{\bullet}(0)=1 ; U^{\bullet}(1)=\frac{1}{2} ; \\
& V^{\bullet}(0)=0 ; \quad V^{\bullet}(1)=\frac{1}{2} ; \\
& G^{\bullet}(0)=0 ; \quad G^{\bullet}(1)=\frac{1}{2} ; \\
& H^{\bullet}(0)=0 ; \quad H^{\bullet}(1)=\frac{1}{2}
\end{aligned}
$$

Поэтому, в частности, получаем:

$$
\begin{gathered}
Q_{z}(0, N)=U(0,1)-G(0,1) ; \\
Q_{z}(1, N)=1-U(0,1) .
\end{gathered}
$$

\section{Вариант $a \leq L \leq 2 a$}

$$
\begin{gathered}
Q(a, L, N)=1-\frac{1}{2}\left(\frac{2 a-L}{a}\right)^{2}+ \\
+\sum_{j=0}^{n} \frac{\delta(j)(N-1) !}{j !(N-1-j) !} \int_{-a}^{a-L}\left(\frac{1}{2}\right)^{j}\left(1+\frac{x}{a}\right)^{2 j} \times \\
\times\left(1-\frac{1}{2}\left(1+\frac{x}{a}\right)^{2}\right)^{N-1-j} \frac{a-L-x}{a^{2}} d x .
\end{gathered}
$$

В этой формуле аналогично первое слагаемое соответствует случаям, когда измерения от дрейфующего датчика оказались в интервале от $-a-L$ до $-a$; сумма интегралов соответствует только одному возможному варианту пересечений интервалов значений измеряемой величины, когда восходящий участок исходной функции плотности распределения пересекается с нисходящим участком смещенной функции плотности распределения.

Обозначим $t=x / a ; z=L / a$. При $1 \leq z \leq 2$ перепишем полученную формулу: 


$$
\begin{gathered}
Q(z, N)=1-\frac{1}{2}(2-z)^{2}+ \\
+\sum_{j=0}^{n} \frac{\delta(j)(N-1) !}{j !(N-1-j) !} \int_{-1}^{1-z}\left(\frac{1}{2}\right)^{j}(1+t)^{2 j} \times \\
\times\left(1-\frac{1}{2}(1+t)^{2}\right)^{N-1-j}(1-z-t) d t .
\end{gathered}
$$

Распишем сумму как две отдельные суммы:

$$
\begin{gathered}
Q(z, N)=1-\frac{1}{2}(2-z)^{2}+ \\
+(2-z)\left(\frac{1}{2}\right)^{N-1} \sum_{j=0}^{n} \frac{\delta(j)(N-1) !}{j !(N-1-j) !} \times \\
\times \int_{-1}^{1-z}(1+t)^{2 j}\left(2-(1+t)^{2}\right)^{N-1-j} d t+ \\
+\left(\frac{1}{2}\right)^{N-1} \sum_{j=0}^{n} \frac{\delta(j)(N-1) !}{j !(N-1-j) !} \times \\
\times \int_{-1}^{1-z}(1+t)^{2 j}\left(2-(1+t)^{2}\right)^{N-1-j}(1+t) d t .
\end{gathered}
$$

Сделаем замену переменной интегрирования путем смещения на единицу:

$$
\begin{gathered}
Q(z, N)=1-\frac{1}{2}(2-z)^{2}+ \\
+(2-z)\left(\frac{1}{2}\right)^{N-1} \sum_{j=0}^{n} \frac{\delta(j)(N-1) !}{j !(N-1-j) !} \times \\
\times \int_{0}^{2-z} t^{2 j}\left(2-t^{2}\right)^{N-1-j} d t+ \\
+\left(\frac{1}{2}\right)^{N-1} \sum_{j=0}^{n} \frac{\delta(j)(N-1) !}{j !(N-1-j) !} \times \\
\times \int_{0}^{2-z} t^{2 j}\left(2-t^{2}\right)^{N-1-j} t d t .
\end{gathered}
$$

Формула приобретает аналогичный унифицированный вид:

$$
\begin{gathered}
Q(z, N)=1-\frac{1}{2}(2-z)^{2}+ \\
+(2-z) U(0,2-z)-V(0,2-z) .
\end{gathered}
$$

В частности:

$$
\begin{gathered}
Q(1, N)=\frac{1}{2}+U(0,1)-V(0,1) ; \\
Q(2, N)=1 .
\end{gathered}
$$

Запишем также производную:

$$
\begin{gathered}
Q_{z}(z, N)=(2-z)-U(0,2-z)- \\
-(2-z) U^{\bullet}(2-z)+V^{\bullet}(2-z) .
\end{gathered}
$$

В частности:

$$
\begin{gathered}
Q_{z}(1, N)=1-U(0,1)-U^{\bullet}(1)+V^{\bullet}(1)= \\
=1-U(0,1) ; \\
Q_{z}(2, N)=V^{\bullet}(0)=0 .
\end{gathered}
$$

Сравнивая (37) и (38), обнаруживаем, что при $z=1$ производная $Q_{z}(z, N)$ обладает свойством непрерывности, несмотря на угловатость функции плотности распределения.

Вычисление интегралов четырех типов. Таким образом, задача сводится к вычислению интегралов. Точнее говоря, имеем дело с суммами интегралов. При этом обнаруживаем возможность успешно выполнять интегрирование именно благодаря суммированию.

Вычисление U. В [11, с. 72] указаны несколько вариантов преобразования интеграла типа (30). В каждом варианте предлагается повышение, понижение или сохранение степени каждого из множителей подынтегрального выражения. Здесь мы выберем наиболее очевидный вариант «по частям», обеспечивающий повышение степени одного множителя и понижение степени другого множителя, тем самым достигая подобия с соседним членом суммируемого ряда, имеющим меньшее значение индекса $j$.

При $j>0$ получается следующая формула преобразования:

$$
\begin{gathered}
\frac{\delta(j)(N-1) !}{j !(N-1-j) !} \int_{t_{1}}^{t_{2}} t^{2 j}\left(2-t^{2}\right)^{N-1-j} d t= \\
=-\frac{\delta(j)(N-1) !}{2 j !(N-1-(j-1)) !} \times \\
\times\left[t^{2 j-1}\left(2-t^{2}\right)^{N-1-(j-1)}\right]_{t_{1}}^{t_{2}}+ \\
\quad+\frac{\delta(j)(2 j-1)(N-1) !}{2 j !(N-1-(j-1)) !} \times \\
\times \int_{t_{1}}^{t_{2}} t^{2(j-1)}\left(2-t^{2}\right)^{N-1-(j-1)} d t .
\end{gathered}
$$

Тем самым в порядке убывания индекса $j$ удается избавиться от всех знаков интеграла, кроме начального, которому соот- 
ветствует $j=0$.

Требуемая сумма интегралов приобретает вид суммы, вполне удобной для программирования:

$$
\begin{aligned}
& U\left(t_{1}, t_{2}\right)=\frac{1}{2^{N-1}}\left(R(0) \int_{t_{1}}^{t_{2}}\left(2-t^{2}\right)^{N-1} d t-\right. \\
& \left.-\sum_{j=1}^{n} \frac{R(j)(N-1) !}{2 j !(N-j) !}\left[t^{2 j-1}\left(2-t^{2}\right)^{N-j}\right]_{t_{1}}^{t_{2}}\right) .
\end{aligned}
$$

Здесь обозначен множитель, вычисляемый рекурсивно:

$$
\begin{gathered}
R(n)=\delta(n) ; \\
R(j-1)=\delta(j-1)+R(j)\left(1-\frac{1}{2 j}\right) .
\end{gathered}
$$

Для слагаемого, которому в выражениях (30) и (39) соответствует $j=0$, нетрудно построить отдельный процесс преобразования интеграла в сумму:

$$
\begin{gathered}
\int_{t_{1}}^{t_{2}}\left(2-t^{2}\right)^{N-1} d t= \\
=\sum_{j=0}^{N-1}(-1)^{j} 2^{N-1-j} \frac{(N-1) !}{j !(N-1-j) !} \int_{t_{1}}^{t_{2}} t^{2 j} d t= \\
=\sum_{j=0}^{N-1}(-1)^{j} 2^{N-1-j} \frac{(N-1) !}{j !(N-1-j) !(2 j+1)}\left[t^{2 j+1}\right]_{t_{1}}^{t_{1}} .
\end{gathered}
$$

Есть и другой вариант преобразования этого интеграла в сумму:

$$
\begin{gathered}
\int_{t_{1}}^{t_{2}}\left(2-t^{2}\right)^{N-1} d t= \\
=\int_{t_{1}}^{t_{2}}(\sqrt{2}-t)^{N-1}(\sqrt{2}+t)^{N-1} d t= \\
=\frac{1}{N}\left[(\sqrt{2}-t)^{N-1}(\sqrt{2}+t)^{N}\right]_{t_{1}}^{t_{2}}+ \\
+\frac{N-1}{N} \int_{t_{1}}^{t_{2}}(\sqrt{2}-t)^{N-2}(\sqrt{2}+t)^{N} d t= \\
=\frac{1}{N}\left[(\sqrt{2}-t)^{N-1}(\sqrt{2}+t)^{N}\right]_{t_{1}}^{t_{2}}+ \\
+\frac{N-1}{N(N+1)}\left[(\sqrt{2}-t)^{N-2}(\sqrt{2}+t)^{N+1}\right]_{t_{1}}^{t_{2}}+ \\
+\frac{(N-1)(N-2)}{N(N+1)} \int_{t_{1}}^{t_{2}}(\sqrt{2}-t)^{N-3}(\sqrt{2}+t)^{N+1} d t=
\end{gathered}
$$

Продолжая аналогично, заметим, что числитель и знаменатель, будучи произведениями убывающих и возрастающих чисел, представимы в виде отношений факториалов. Такими последовательными шагами получается сумма и остается элементарный интеграл, который можно рассматривать как слагаемое с индексом $N-1$ :

$$
\begin{gathered}
=\sum_{j=0}^{N-2} \frac{(N-1) !(N-1) !}{(N+j) !(N-1-j) !} \times \\
\times\left[(\sqrt{2}-t)^{N-1-j}(\sqrt{2}+t)^{N+j}\right]_{t_{1}}^{t_{2}}+ \\
+\frac{(N-1) !(N-1) !}{(2 N-2) !} \int_{t_{1}}^{t_{2}}(\sqrt{2}+t)^{2 N-2} d t .
\end{gathered}
$$

В итоге получаем:

$$
\begin{gathered}
\int_{t_{1}}^{t_{2}}\left(2-t^{2}\right)^{N-1} d t=\sum_{j=0}^{N-1} \frac{(N-1) !(N-1) !}{(N+j) !(N-1-j) !} \times \\
\times\left[(\sqrt{2}-t)^{N-1-j}(\sqrt{2}+t)^{N+j}\right]_{t_{1}}^{t_{2}} .
\end{gathered}
$$

Вычисление $V$. В составе суммы (31) при $j>0$ возьмем интеграл «По частям»:

$$
\begin{gathered}
\frac{\delta(j)(N-1) !}{j !(N-1-j) !} \int_{t_{1}}^{t_{2}} t^{2 j}\left(2-t^{2}\right)^{N-1-j} t d t= \\
=-\frac{\delta(j)(N-1) !}{2 j !(N-1-(j-1)) !} \times \\
\times\left[t^{2 j}\left(2-t^{2}\right)^{N-1-(j-1)}\right]_{t_{1}}^{t_{2}}+ \\
\quad+\frac{\delta(j)(N-1) !}{2 j !(N-1-(j-1)) !} \times \\
\times \int_{t_{1}}^{t_{2}} 2 j t^{2 j-1}\left(2-t^{2}\right)^{N-1-(j-1)} d t= \\
=-\frac{\delta(j)(N-1) !}{2 j !(N-j) !}\left[t^{2 j}\left(2-t^{2}\right)^{N-j}\right]_{t_{1}}^{t_{2}}+ \\
+\frac{\delta(j)(N-1) !}{(j-1) !(N-1-(j-1)) !} \times \\
\times \int_{t_{1}}^{t_{2}} t^{2(j-1)}\left(2-t^{2}\right)^{N-1-(j-1)} t d t .
\end{gathered}
$$

Как видно, здесь получается интеграл такого же вида, как и интеграл с индексом $j-1$ среди членов суммируемого ряда (31). Применяя эту формулу последовательно в порядке убывания индекса $j$ и объединяя подобные члены ряда, избавляемся от всех знаков интеграла. При этом интеграл с индексом $j=0$ без рекурсивной зависимости превращается в аналогичный член получа- 
ющегося суммируемого ряда:

$$
\begin{gathered}
V\left(t_{1}, t_{2}\right)=-\left(\frac{1}{2}\right)^{N} \times \\
\times \sum_{j=0}^{n} \frac{\left(\frac{N}{2}-j\right)(N-1) !}{j !(N-j) !}\left[t^{2 j}\left(2-t^{2}\right)^{N-j}\right]_{t_{1}}^{t_{2}} .
\end{gathered}
$$

Появившийся здесь множитель $N / 2-j$ является следствием объединения подобных членов ряда с учетом обозначений (5)-(8).

Существует возможность дальнейшего преобразования:

$$
\begin{gathered}
V\left(t_{1}, t_{2}\right)=-\left(\frac{1}{2}\right)^{N+1} \times \\
\times \sum_{j=0}^{n} \frac{(N-2 j)(N-1) !}{j !(N-j) !}\left[t^{2 j}\left(2-t^{2}\right)^{N-j}\right]_{t_{1}}^{t_{2}}=,
\end{gathered}
$$

рассматривая множитель $N-2 j$, как два слагаемых $N-j$ и $j$ :

$$
\begin{aligned}
& =\frac{1}{2^{N+1}} \sum_{j=1}^{n} \frac{(N-1) !}{(j-1) !(N-j) !}\left[t^{2 j}\left(2-t^{2}\right)^{N-j}\right]_{t_{1}}^{t_{2}}- \\
& -\frac{1}{2^{N+1}} \sum_{j=0}^{n} \frac{(N-1) !}{j !(N-1-j) !}\left[t^{2 j}\left(2-t^{2}\right)^{N-j}\right]_{t_{1}}^{t_{2}}=,
\end{aligned}
$$

переобозначая индекс $j$ путем смещения на единицу:

$$
\begin{aligned}
& =\frac{1}{2^{N+1}} \sum_{j=0}^{n} \frac{(N-1) !}{j !(N-1-j) !}\left[t^{2+2 j}\left(2-t^{2}\right)^{N-1-j}\right]_{t_{1}}^{t_{2}}- \\
& -\frac{1}{2^{N+1}} \sum_{j=0}^{n} \frac{(N-1) !}{j !(N-1-j) !}\left[t^{2 j}\left(2-t^{2}\right)^{N-j}\right]_{t_{1}}^{t_{2}}=,
\end{aligned}
$$

объединяя попарно слагаемые двух сумм, выписывая отдельно слагаемое с индексом $n$, которое осталось без пары:

$$
\begin{gathered}
=\frac{1}{2^{N}} \sum_{j=0}^{n-1} \frac{(N-1) !}{j !(N-1-j) !} \times \\
\times\left[\left(t^{2}-1\right) t^{2 j}\left(2-t^{2}\right)^{N-1-j}\right]_{t_{1}}^{t_{2}}- \\
-\frac{1}{2^{N+1}} \frac{(N-1) !}{n !(N-1-n) !}\left[t^{2 n}\left(2-t^{2}\right)^{N-1-n}\right]_{t_{1}}^{t_{2}} .
\end{gathered}
$$

В этом виде формула полезна тем, что при $t=1$ избавляет нас от необходимости вычислять сумму. Поэтому, в частности, получаем:

$$
V(0,1)=\frac{1}{2}-\left(\frac{1}{2}\right)^{N+1} \frac{(N-1) !}{n !(N-1-n) !} .
$$

Вычисление $G$.

$$
\begin{gathered}
G\left(t_{1}, t_{2}\right)=\left(\frac{1}{2}\right)^{N-1} \times \\
\times \sum_{j=0}^{n} \frac{\delta(j)(N-1) !}{j !(N-1-j) !} \int_{t_{1}}^{t_{2}} t^{2(N-1-j)}\left(2-t^{2}\right)^{j} d t .
\end{gathered}
$$

В составе суммы (32) при $j>0$ возьмем интеграл «по частям»:

$$
\begin{aligned}
& \frac{\delta(j)(N-1) !}{j !(N-1-j) !} \int_{t_{1}}^{t_{2}} t^{2(N-1-j)}\left(2-t^{2}\right)^{j} d t= \\
& =\frac{\delta(j)(N-1) !}{j !(N-1-j) !}\left[\frac{t^{2(N-1-j)+1}\left(2-t^{2}\right)^{j}}{2(N-1-j)+1}\right]_{t_{1}}^{t_{2}}+ \\
& +\frac{\delta(j)(N-1) !}{j !(N-1-j) !} \int_{t_{1}}^{t_{2}} \frac{2 j}{2(N-1-j)+1} \times \\
& \times t^{2(N-1-(j-1))}\left(2-t^{2}\right)^{j-1} d t= \\
& =\frac{\delta(j)(N-1) !}{2 j !(N-1-j) !}\left[\frac{t^{2(N-1-j)+1}\left(2-t^{2}\right)^{j}}{(N-1-(j-1))-\frac{1}{2}}\right]_{t_{1}}^{t_{2}}+ \\
& +\frac{\delta(j)(N-1) !}{j !(N-1-j) !} \int_{t_{1}}^{t_{2}} \frac{j}{(N-1-(j-1))-\frac{1}{2}} \times \\
& \times t^{2(N-1-(j-1))}\left(2-t^{2}\right)^{j-1} d t= \\
& =\frac{\delta(j)(N-1) !}{2 j !(N-1-(1-j)) !} \times \\
& \times\left[\frac{t^{2(N-1-j)+1}\left(2-t^{2}\right)^{j}}{1-\frac{1}{2(N-1-(j-1))}}\right]_{t_{1}}^{t_{2}}+ \\
& +\frac{\delta(j)(N-1) !}{(j-1) !(N-1-(j-1)) !} \times \\
& \times \int_{t_{1}}^{t_{2}} \frac{t^{2(N-1-(j-1))}\left(2-t^{2}\right)^{j-1}}{1-\frac{1}{2(N-1-(j-1))}} d t .
\end{aligned}
$$

Как видно, получается интеграл, подобный интегралу с меньшим значением индекса $j$. Поэтому удобно применять эту формулу последовательно в порядке убывания индекса $j$. Получается сумма, в которой остается только один простой интеграл, которому соответствует $j=0$. Этот интеграл подпадает под общую формулу каждого 
члена суммируемого ряда, имеющего следующий итоговый вид:

$$
\begin{aligned}
G\left(t_{1}, t_{2}\right) & =\left(\frac{1}{2}\right)^{N} \sum_{j=0}^{n} \frac{R(j)(N-1) !}{j !(N-j) !} \times \\
\times & {\left[t^{2(N-j)-1}\left(2-t^{2}\right)^{j}\right]_{t_{1}}^{t_{2}} . }
\end{aligned}
$$

Здесь обозначен множитель, вычисляемый рекурсивно:

$$
\begin{gathered}
R(n)=\frac{\delta(n)}{1-\frac{1}{2(N-n)}} ; \\
R(j)=\frac{\delta(j)+R(j+1)}{1-\frac{1}{2(N-j)}}
\end{gathered}
$$

Вычисление Н. В составе суммы (33) при $j>0$ возьмем интеграл «по частям»:

$$
\begin{gathered}
\frac{\delta(j)(N-1) !}{j !(N-1-j) !} \int_{t_{1}}^{t_{2}} t^{2(N-1-j)}\left(2-t^{2}\right)^{j} t d t= \\
=\frac{\delta(j)(N-1) !}{j !(N-1-j) !}\left[\frac{t^{2(N-1-j)+2}\left(2-t^{2}\right)^{j}}{2(N-1-j)+2}\right]_{t_{1}}^{t_{2}}+ \\
+\frac{\delta(j)(N-1) !}{j !(N-1-j) !} \int_{t_{1}}^{t_{2}} \frac{2 j}{2(N-1-j)+2} \times \\
=\frac{\delta(j)(N-1) !}{2 j !(N-j) !}\left[t^{2(N-j)}\left(2-t^{2}\right)^{j}\right]_{t_{1}}^{t_{2}}+ \\
+\frac{\delta(j)(N-1) !}{(j-1) !(N-1-(j-1)) !} \times \\
\quad \times \int_{t_{1}}^{t_{2}} t^{2(N-1-(j-1))}\left(2-t^{2}\right)^{j-1} t d t .
\end{gathered}
$$

Получился интеграл такого же вида, как и интеграл с индексом $j-1$ среди членов суммируемого ряда (33). Применяя эту формулу последовательно, в порядке убывания индекса $j$, и объединяя подобные члены ряда, избавляемся от всех знаков интеграла. При этом интеграл с индексом $j=0$ без рекурсивной зависимости превращается в аналогичный член получающегося суммируемого ряда:

$$
\begin{aligned}
H\left(t_{1}, t_{2}\right)= & \left(\frac{1}{2}\right)^{N} \sum_{j=0}^{n}\left(\frac{N}{2}-j\right) \frac{(N-1) !}{j !(N-j) !} \times \\
& \times\left[t^{2(N-j)}\left(2-t^{2}\right)^{j}\right]_{t_{1}}^{t_{2}} .
\end{aligned}
$$

Появившийся здесь множитель $N / 2-j$ является следствием объединения подобных членов ряда с учетом обозначений (5)-(8).

Существует возможность дальнейших преобразований. Этот множитель нам нужен как два слагаемых, позволяющих рассматривать две суммы:

$$
\begin{aligned}
& H\left(t_{1}, t_{2}\right)=\left(\frac{1}{2}\right)^{N+1} \sum_{j=0}^{n}(N-j-j) \frac{(N-1) !}{j !(N-j) !} \times \\
& =\left(\frac{1}{2}\right)^{N+1} \sum_{j=0}^{n} \frac{(N-1) !}{j !(N-1-j) !}\left[t^{2(N-j)}\left(2-t^{2}\right)^{j}\right]_{t_{1}}^{t_{2}}- \\
& -\left(\frac{1}{2}\right)^{N+1} \sum_{j=1}^{n} \frac{(N-1) !}{(j-1) !(N-j) !}\left[t^{2(N-j)}\left(2-t^{2}\right)^{j}\right]_{t_{1}}^{t_{2}}=
\end{aligned}
$$

Во второй сумме выполняем замену индекса $j$ путем смещения на единицу:

$$
\begin{aligned}
=\left(\frac{1}{2}\right)^{N+1} & \sum_{j=0}^{n} \frac{(N-1) !}{j !(N-1-j) !}\left[t^{2(N-j)}\left(2-t^{2}\right)^{j}\right]_{t_{1}}^{t_{2}}- \\
- & \left(\frac{1}{2}\right)^{N+1} \sum_{j=0}^{n-1} \frac{(N-1) !}{j !(N-1-j) !} \times \\
& \times\left[t^{2(N-1-j)}\left(2-t^{2}\right)^{j+1}\right]_{t_{1}}^{t_{2}}=
\end{aligned}
$$

Попарно объединяем все члены двух сумм, кроме $n$-го члена, оставшегося без пары:

$$
\begin{aligned}
=\left(\frac{1}{2}\right)^{N+1} & \frac{(N-1) !}{n !(N-1-n) !}\left[t^{2(N-n)}\left(2-t^{2}\right)^{n}\right]_{t_{1}}^{t_{2}}+ \\
& +\left(\frac{1}{2}\right)^{N} \sum_{j=0}^{n-1} \frac{(N-1) !}{j !(N-1-j) !} \times \\
& \times\left[\left(t^{2}-1\right) t^{2(N-1-j)}\left(2-t^{2}\right)^{j}\right]_{t_{1}}^{t_{2}} .
\end{aligned}
$$

В этом виде формула полезна тем, что при $t=1$ избавляет нас от необходимости вычислять сумму. Поэтому, в частности, получаем:

$$
H(0,1)=\left(\frac{1}{2}\right)^{N+1} \frac{(N-1) !}{n !(N-1-n) !} .
$$

В качестве проверки правильности формул заметим, что (40) и (41) в сумме соответствуют тождествам (35) и (36).

\section{Графики зависимости $Q(z, N)$}

В результате вычислений по получен- 


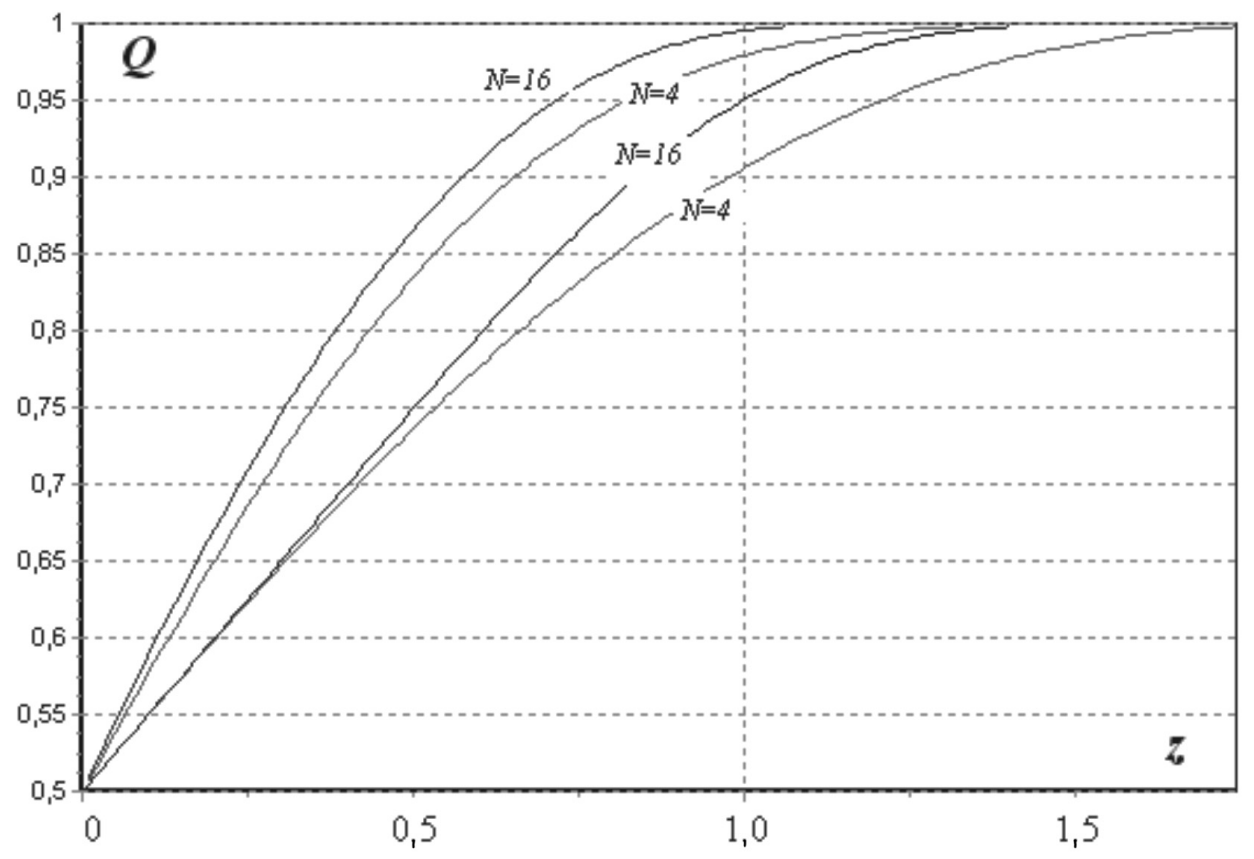

Рис. 1. Графики дисбаланса для равномерного и треугольного законов распределений

ным формулам построены графики, представленные на рис. 1.

Все графики дисбаланса для равномерного (прямоугольного) распределения имеют одинаковый тангенс наклона в точке $(0,05)$. Графики дисбаланса для треуголь- ного распределения сразу же разветвляются из начальной точки и идут более круто.

Чтобы наглядно изобразить эти два семейства кривых и вместе с тем не загромождать рисунок, от каждого семейства выбраны по две линии для $N=4$ и $N=16$.

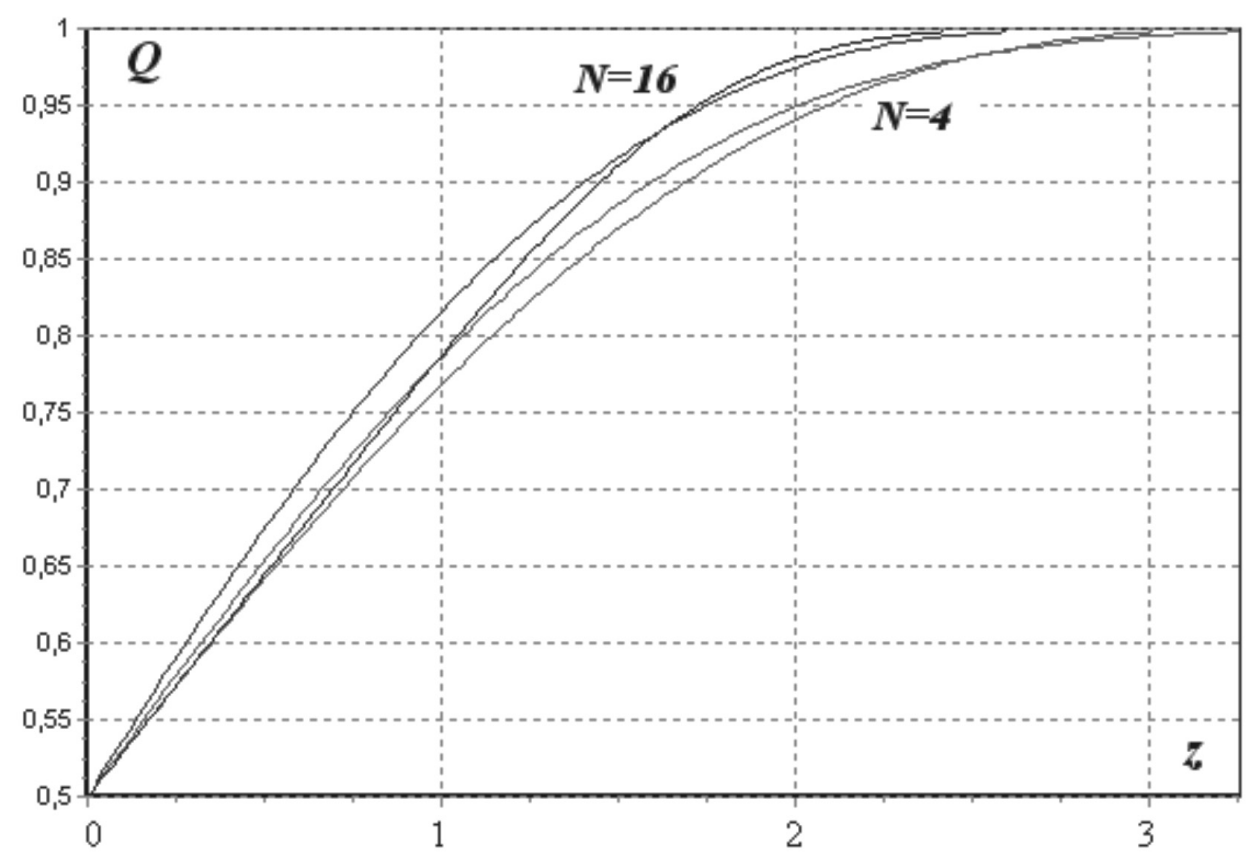

Рис. 2. Нормированные графики дисбаланса для равномерного и треугольного законов распределений 
Эффект нейтральности серединного элемента проявляется всегда, а не только для (22) и (23).

Рассмотренные два типа распределения являются идеализированными моделями. Но они полезны тем, что могут рассматриваться в качестве границ для многих реальных форм распределения. Очевидно, что и полученные графики дисбаланса приобретают роль границ.

На рис. 2 эти же линии представлены нормированными по горизонтали по среднеквадратическому отклонению $\sigma$ (с учетом выражений (10) и (29)) по формуле $z=L / \sigma$. Иначе говоря, для треугольного распределения в сравнении с равномерным распределением горизонтальный масштаб расширен с коэффициентом $\sqrt{2}$. Как видно, при этом достигается близкое сходство линий дисбаланса для двух типов распределения. Поэтому уместно предположить, что и для многих других типов распределения, если аналогично масштабировать по среднеквадратическому отклонению, получающиеся приближенные графики будут приемлемыми. Однако эта тема остается для следующих статей.

Представленные формулы и результаты вычислений предоставляют зависимость дисбаланса от величины дрейфа одного измерителя в качестве основы для динамической корректировки коэффициентов чувствительности однотипных датчиков.

\section{СПИСОК ЛИТЕРАТУРЫ}

1. Тайманов Р.Е., Сапожникова К.В. Метрологический самоконтроль датчиков // Сб. трудов II Росс. конф. Технические и программные средства систем управления, контроля и измерения (теория, методы, алгоритмы, исследования и разработки). М.: ИПУ РАН, 2010. Датчики в системах управления, контроля и измерения [Электронный ресурс]/ URL: http://cmm.ipu.ru/ node/15; http://cmm.ipu.ru/proc

2. Dahir Insaat. Combat Robot (in Russian) [Электронный ресурс]/ URL: http://fullreels.com/ en/video/UZbqsYYapW4/Dahir-Insaat-CombatRobot-in-Russian (Дата обращения: 11.07.2016).

3. Власенко А.Н., Демченков В.П., Лапин О.Е., Лопота В.А., Никуленков К.П., Шелепков Е.А., Юдин В.И. Устройство для измерения потоков фотонного излучения // Патент РФ на изобретение № 2299450. Приоритет 20.05.2007.

4. Измеритель мощности дозы и дифференциальных потоков гамма-излучения ИМД-24 [Электронный ресурc]/ URL: http://www.rtc.ru/ index.php/sredstva-radiatsionnogo-kontrolya/imd24 (Дата обращения: 04.04.2016).

5. Аркадьев В.Б., Голубева О.А., Ильин А.С., Лапин О.Е. Особенности программного обеспе- чения измерителя мощности дозы и дифференциальных потоков гамма-излучения: Презентация. 28.02.2011 [Электронный ресурс]/ URL: http://www.atomic-energy.ru/presentations/19074 (Дата обращения: 12.01.2016).

6. НПФ «Консенсус». Каталог счетчиков регистрации излучений [Электронный ресурс]/ URL: http://consensus-group.ru/katalog (Дата обращения: 04.04.2016).

7. Бойко А.Ю., Васильев А.В. Мобильный многоцелевой робототехнический комплекс // Труды междунар. науч.-техн. конф. Экстремальная робототехника. СПб.: ЦНИИ РТК, 2014. C. $46-49$.

8. Дэйвид Г. Порядковые статистики. М.: Наука. Гл. ред. Физматлит, 1979. 336 с.

9. Гильбо Е.П., Челпанов И.Б. Обработка сигналов на основе упорядоченного выбора (мажоритарное и близкие к нему преобразования). М.: Советское радио, 1976. 344 с.

10. Виленкин Н.Я., Виленкин А.Н., Виленкин П.А. Комбинаторика. М.: ФИМА, МЦНМО, 2006. $400 \mathrm{c}$.

11. Градштейн И.С., Рыжик И.М. Таблицы интегралов, сумм, рядов и произведений. 5-е изд. М.: Наука, 1971.

\section{REFERENCES}

1. Tajmanov R.E., Sapozhnikova K.V. Metrologicheskiy samokontrol datchikov [Metrological self-checking of sensors]. Tehnicheskie i programmnye sredstva sistem upravlenija, kontrolja $i$ izmerenija (teorija, metody, algoritmy, issledovanija $i$ razrabotki) [Proceedings of the 2nd Russian Conference with the International Participation,
Technical and Software of Control Systems, Control and Measurement (the theory, methods, algorithms, researches and development)]. Moscow, IPU RAN, Oct. 18-20, 2010. Datchiki v sistemah upravlenija, kontrolja $i$ izmerenija [Sensors in control and measurement systems]. Available: http://cmm.ipu. ru/node/15; http://cmm.ipu.ru/proc/index.html 
(Accessed: 12.02.2016). (rus)

2. Dahir Insaat Combat Robot. Available: http://fullreels.com/en/video/UZbqsYYapW4/ Dahir-Insaat-Combat-Robot-in-Russian (Accessed: 11.07.2016). (rus)

3. Vlasenko A.N., Demchenkov V.P., Lapin O.E., Lopota V.A., Nikulenkov K.P., Shelepkov E.A., Judin V.I. Ustrojstvo dlja izmerenija potokov fotonnogo izluchenija. Patent RF no. 2299450 [Patent for the Device for Measurement of Streams of Photon Radiation]. Prioritet 20.05.2007. (rus)

4. Izmeritel moschnosti dozyi $i$ differentsialnyih potokov gamma-izlucheniya IMD-24 [Measuring instrument of power of a dose and differential streams of gamma radiation IMD-24]. Available: http:// www.rtc.ru/index.php/sredstva-radiatsionnogokontrolya/imd-24 (Accessed: 04.04.2016). (rus)

5. Arkad'ev V.B., Golubeva O.A., Ilyin A.S., Lapin O.E. Osobennosti programmnogo obespecheniya izmeritelya moschnosti dozyi $i$ differentsialnyih potokov gamma-izlucheniya [Features of the software of the measuring instrument of power of a dose and differential streams of gamma radiation, presentation]. Feb. 28, 2011. Available: http://www.atomic-energy. ru/presentations/19074 (Accessed: 12.02.2016). (rus)
6. NPF «Konsensus». Katalog schetchikov registratsii izlucheniy [Catalog of counters of registration of radiations]. Available: http://consensus-group.ru/ katalog (Accessed: 06.04.2016). (rus)

7. Boyko A.Yu., Vasiliev A.V. Multi-purpose mobile robotic complex. Proceedings of the International Scientific and Technological Conference Exteme robotics, State Scientific Center for Robotics and Technical Cybernetics, Oct. 1-2, 2014, St. Petersburg, Russia, Pp. 46-49. (rus)

8. David H., Nagaraja H. Order statistics, 3d ed., Wiley, 2003.

9. Gil'bo E.P., Chelpanov I.B. Obrabotka signalov na osnove uporjadochennogo vybora (mazhoritarnoe $i$ blizkie $k$ nemu preobrazovanija) [Processing of signals on the basis of the ordered choice (majority and other transformations)]. Moscow: Sovetskoe radio Publ., 1976, 344 p. (rus)

10. Vilenkin N.Ja., Vilenkin A.N., Vilenkin P.A. Kombinatorika [Combinatorics]. Moscow: FIMA and Moscow Center of Continuous Mathematical Education Publ., 2006, 400 p. (rus)

11. Gradshtejn I.S., Ryzhik I.M. Tablicy integralov, summ, rjadov $i$ proizvedenij [Tables of integrals, sums and serieses]. Moscow: Nauka Publ., 1971. (rus)

ИЛЬИН Анатолий Степанович - старший научный сотрудник Центрального научно-исследовательского и опытно-конструкторского института робототехники и технической кибернетики, кандидат технических наук.

194064, Россия, Санкт-Петербург, Тихорецкий пр., д. 21.

E-mail: TOLY@RTC.RU

ILYIN Anatoliy S. State Scientific Center for Robotics and Technical Cybernetics (RTC).

194064, Tikhoretsky Ave. 21, St. Petersburg, Russia.

E-mail: TOLY@RTC.RU 